\title{
Crecimiento y heterogeneidad del capital humano: efectos de la expansión de la educación superior en el aumento del ingreso en los municipios del Brasil
}

\author{
Leonardo Andrade Rocha, Napiê Galvê Araújo Silva, Carlo Alano \\ Soares de Almeida, Denison Murilo de Oliveira y Kaio César Fernandes
}

\section{Resumen}

En el presente estudio se analizan los efectos de la expansión de la mano de obra altamente calificada (educación superior) en el aumento del ingreso per cápita de los municipios brasileños. Los resultados muestran que el incremento de la mano de obra con estudios superiores se traduce en un mayor crecimiento en los municipios más desarrollados y con menores costos de oportunidad, medido por las diferencias salariales. La reducción de las desigualdades regionales promovida por la difusión de la educación superior en los últimos 15 años se vuelve sostenible si el aumento de la oferta de competencias está acompañado por la ampliación de la demanda, de manera que se reducen los costos de oportunidad entre los factores y se incrementa la "prima de calificación".

\section{Palabras clave}

Recursos humanos, mano de obra, trabajadores calificados, gobierno municipal, ingresos, desigualdades regionales, crecimiento económico, Brasil

Clasificación JEL

E24, O43, Q55, R11

\section{Autores}

Leonardo Andrade Rocha es Doctor en Desarrollo Económico y Profesor en el Centro de Ciencias Sociales Aplicadas y Humanas (CCSAH) de la Universidad Federal Rural do Semi-Árido (UFERSA), Brasil. Correo electrónico: leonardo.rocha@ufersa.edu.br. Napiê Galvê Araújo Silva es Doctor en Ciencias Sociales y Profesor en el Centro de Ciencias Sociales Aplicadas y Humanas (CCSAH) de la Universidad Federal Rural do Semi-Árido (UFERSA), Brasil. Correo electrónico: pie@ufersa.edu.br.

Carlos Alano Soares de Almeida es Doctor en Administración y Profesor en el Centro de Ciencias Sociales Aplicadas y Humanas (CCSAH) de la Universidad Federal Rural do Semi-Árido (UFERSA), Brasil. Correo electrónico: alano@ufersa.edu.br.

Denison Murilo de Oliveira es Doctor en Administración y Profesor en el Centro de Ciencias Sociales Aplicadas y Humanas (CCSAH) de la Universidad Federal Rural do Semi-Árido (UFERSA), Brasil. Correo electrónico: denison@ufersa.edu.br.

Kaio César Fernandes es Doctor en Ciencias Sociales y Profesor en el Centro de Ciencias Sociales Aplicadas y Humanas (CCSAH) de la Universidad Federal Rural do Semi-Árido (UFERSA), Brasil. Correo electrónico: kaio@ufersa.edu.br. 


\section{Introducción}

La importancia del capital humano para el desarrollo y la reducción de las desigualdades económicas se ha destacado en numerosos estudios (Barro, 2001; Barro y Sala-i-Martin, 1992; Becker y Woessmann, 2009; Becker, Hornung y Woessmann, 2011; Benhabib y Spiegel, 1994). Esto deriva de la necesidad del conocimiento incorporado en los individuos a medida que la economía avanza en su desarrollo. El conocimiento es importante, tanto para absorber las técnicas de producción existentes como para crear una nueva generación de tecnologías (Benhabib y Spiegel, 2005). El proceso de acumulación de capital humano es histórico y se ha asociado con un importante factor de divergencia económica en el mundo (Acemoglu, Gallego y Robinson, 2014).

Si bien en esos estudios se ha descrito la importancia del capital humano en el desarrollo, en algunas investigaciones recientes se ha presentado su carácter heterogéneo en el mismo desarrollo (Vandenbussche, Aghion y Meghir, 2006; Ang, Madsen e Islam, 2011; Ott y Soretz, 2011; Basu y Mehra, 2014; Madsen, 2014). Existen diferentes tipos de capital humano que se complementan con el patrón tecnológico vigente en la economía (Caselli y Coleman, 2006). Así, se distinguen distintos tipos de capital humano que se asignan a diferentes actividades, de manera que las divergencias económicas pueden estar relacionadas con las diferencias en su composición entre las economías (Basu y Mehra, 2014).

En la realidad brasileña, los últimos 15 años se caracterizaron por una importante expansión de la educación superior. Esta permitió la formación de una reserva de mano de obra calificada para satisfacer los requisitos de la demanda ante las transformaciones económicas en curso en el país. Estos cambios fueron impulsados internamente, por medio de políticas orientadas a la reducción de las desigualdades regionales, y externamente, conforme al creciente patrón de competencia internacional (Mancebo, Vale y Martins, 2015).

Según datos del Censo de Educación Superior (INEP, 2016), el número de matrículas en la educación superior en el Brasil aumentó de 2.694.245 en 2000 a 8.027.297 en 2015¹. Se trata de un crecimiento de aproximadamente el $198 \%$ en el período mencionado, que evidencia una importante estrategia en la política nacional de educación del país.

Sin embargo, esa expansión no siempre buscó adecuar el crecimiento de la oferta de trabajo calificada a los avances en la demanda. Esta alusión puede encontrarse en investigaciones recientes, en especial Cury (2014), Mancebo, Vale y Martins (2015), Lima (2012) y Costa, Costa y Barbosa (2013). De acuerdo con Cury (2014), en los últimos 20 años se produjeron importantes cambios en la educación superior del país, en particular su expansión mediante una "alianza" entre instituciones públicas y privadas. No obstante, dicha expansión se ha centralizado en importantes mecanismos de financierización y creación de oligopolios dentro del sector, que regularon parte del proceso y comprometieron considerablemente su calidad. La expansión fue orientada con una mayor participación del sector privado en relación con el sector público (Mancebo, Vale y Martins, 2015), de manera que el proceso se realizó y sustentó a lo largo de los últimos años mediante importantes transferencias de recursos públicos del país (Lima, 2012; Costa, Costa y Barbosa, 2013). Además, a lo largo de este proceso se observaron algunos aspectos sociales relativos al acceso a nuevas oportunidades como consecuencia de la expansión de la educación superior, que garantizaron una mayor equidad en términos de oportunidades y favorecieron una mayor igualdad en el acceso al mercado de trabajo, constituyendo un eslabón esencial en la integración social (Felicetti, Cabrera y Costa-Morosini, 2014, pág. 36).

\footnotetext{
1 Véase [en línea] http://portal.inep.gov.br/web/guest/sinopses-estatisticas-da-educacao-superior.
} 
Este aspecto es relevante para el escenario macroeconómico, pues la política apunta a asignar recursos limitados para obtener el máximo bienestar de la sociedad. Desde la perspectiva presupuestaria de cada país, la planificación de la política económica supone la toma de decisiones con el fin de asignar los recursos de manera eficiente. El objetivo de esta asignación es aproximar los rendimientos sociales a los rendimientos privados por el uso de los recursos.

Al igual que en los trabajos de Vandenbussche, Aghion y Meghir (2006), Aghion (2008) y Aghion y otros (2009), en la presente investigación se desarrolló un modelo de crecimiento schumpeteriano, en el que el crecimiento de la productividad está influenciado por dos importantes tipos de actividad: i) la imitación, basada en la actividad de trabajadores poco calificados que implementan tecnologías existentes transferidas desde la frontera tecnológica; y ii) la innovación, que supone la contratación de mano de obra altamente calificada asignada a los departamentos de investigación. Las condiciones de equilibrio del empresario innovador muestran que la demanda de mano de obra calificada y no calificada tiene un peso distinto según la proximidad a la frontera tecnológica. El factor de aproximación a la frontera determina la prima de calificación y la tasa de progreso tecnológico.

A diferencia de otros trabajos, en el presente estudio se emplean las diferencias salariales para medir el efecto de la "distancia de la frontera", pues esta variable afecta directamente los incentivos de asignación entre los factores y está condicionada a las fluctuaciones de proximidad con respecto a la frontera. En las políticas de intervención en el mercado de trabajo, el seguimiento de los ingresos se vuelve más intuitivo y, por lo tanto, más coherente para medir la realidad regional de cada municipio.

Sobre la base de datos del Atlas de Desarrollo Humano en Brasil (PNUD/IPEA/FJP, 2013), a partir de la dimensión político-administrativa más pequeña, se estimó un modelo de regresión lineal que relaciona la reserva de mano obra calificada y el producto interno bruto (PIB) per cápita de los municipios. Controlando el modelo y estimando a partir del método generalizado de momentos, las estimaciones indican que el incremento de la mano de obra con educación superior se traduce en un mayor crecimiento en los municipios más desarrollados y con menores costos de oportunidad, medido por las diferencias salariales.

El debate sobre la composición del capital humano y sus consecuencias en el crecimiento de las economías es todavía reciente, de manera que se han realizado pocos estudios desde una perspectiva interna y regional. Entre ellos se destacan las contribuciones de Zhang y Zhuang (2011), Gennaioli y otros (2013) y Korpi y Clark (2015).

\section{Ambiente económico}

A partir de los aportes de Aghion y Howitt (1998; 2009), el modelo consiste en una economía con un número finito de sectores, cada uno de ellos compuesto por empresarios intermediarios y por una población de trabajadores que ofrecen su fuerza de trabajo en un mercado competitivo. Los trabajadores tienen dotaciones heterogéneas de capital humano de manera que, a nivel agregado, la economía se divide entre $S$ unidades de trabajo altamente calificadas y $U$ unidades poco calificadas, ofrecidas de manera exógena y a una tasa constante en el tiempo. Con un mercado competitivo y la oferta de trabajo definida elásticamente, se obtiene $(s=\widetilde{S}, u=\widetilde{U}) \in \Re_{+}^{2}$, donde $\widetilde{S}, \widetilde{U}$ corresponden, respectivamente, a los niveles de equilibrio de la mano de obra calificada y no calificada en el mercado de trabajo.

Las empresas adoptan estrategias de imitación (implementan las innovaciones de las empresas o los sectores más avanzados) e innovación. Cada empresario decide emplear una combinación equilibrada de las dos estrategias, con miras a obtener los beneficios del monopolio temporal del sector. La decisión de adoptar una práctica más innovadora o imitadora depende de su potencial distancia con respecto a los líderes del sector (empresa con la tecnología de referencia). 
El tiempo es discreto (no continuo) y el producto final de la economía se obtiene utilizando un flujo continuo de insumos intermedios conforme a la función de producción Cobb-Douglas:

$$
Y_{t}=\int_{0}^{1} A_{t}(i)^{1-\alpha} X_{t}(i)^{\alpha} d i \therefore \alpha \in(0,1)
$$

Según la ecuación (1), $x_{t}(i)$ corresponde al flujo de insumos intermedios del sector $i$ en el tiempo $t$. La productividad del sector está dada por el parámetro tecnológico $A_{t}(i)$, que se define por el nivel rezagado $A_{t-1}(i)$ más un "componente estratégico del sector". La definición de este componente se abordará en la próxima sección. El sector del insumo intermedio está monopolizado por la empresa líder, que tiene el monopolio en un corto período de tiempo.

Suponiendo que el sector del producto final es competitivo, cada productor de insumos intermedios se enfrenta a una curva de demanda inversa que define el precio del insumo intermedio:

$$
p_{t}(i)=\frac{\partial Y_{t}}{\partial x_{t}(i)}=\alpha A_{t}(i)^{1-\alpha} x_{t}(i)^{\alpha-1}
$$

En cada sector intermedio, solo un productor intermedio puede producir el producto $i$ con la productividad del sector $A_{t}(i)$ usando el producto final como capital. Este proceso se realizará mediante una tecnología uno a uno (one-for-one technology), siguiendo la especificidad $A_{t}(i): \mathfrak{I} \rightarrow \Omega \therefore Y_{t} \in \mathfrak{I} ; y_{t}(i) \in \Omega$.

En este sentido, el monopolista del sector $i$ elige $x_{t}(i)$, que resuelva el problema de maximización:

$$
\max _{x_{t}(i)}\left\{p_{t}(i) x_{t}(i)-x_{t}(i)\right\}
$$

La solución del problema de maximización (3) genera una función de demanda de equilibrio:

$$
\widehat{x}_{t}(i)=\alpha \frac{2}{1-\alpha} A_{t}(i)
$$

Al sustituir (4) en (3), se obtienen los beneficios de equilibrio del monopolista del sector:

$$
\widehat{\pi}_{t}(i)=\left(p_{t}(i)-1\right) \widehat{x}_{t}(i)=\pi A_{t}(i) ; \pi \equiv\left(\frac{1-\alpha}{\alpha}\right) \alpha \frac{2}{1-\alpha}
$$

A continuación, se definen las fuentes de crecimiento de la productividad y la manera en que se relacionan con la frontera tecnológica.

\section{Fuentes de crecimiento de la productividad}

La dinámica de la tecnología en el sector $i$ puede captarse mediante una función positiva y creciente, $\Phi(\cdot)$, en los siguientes argumentos de la función:

$$
A_{t}(i)=A_{t-1}(i)+\underbrace{\Phi\left(\bar{A}_{t-1}(i)-A_{t-1}(i), A_{t-1}(i), u_{t}(i), s_{t}(i)\right)}_{\text {COMPONENTE ESTRATÉGICO }}
$$

Donde $\bar{A}_{t-1}(i), A_{t-1}(i), s_{t}(i), u_{t}(i)$ corresponden, respectivamente, a la frontera tecnológica del sector $i$ al final del período $t-1$, la productividad del sector $i$ al final del período $t$ - 1 , y a las unidades de mano de obra calificada y no calificada del sector $i$ al final del período $t$. La función $\Phi(\cdot)$ admite ser una función lineal en los componentes de imitación e innovación y no lineal en los recursos humanos. Específicamente, a continuación, se tiene:

$$
A_{t}(i)=A_{t-1}(i)+\left[s_{t}(i)^{\Phi}(\gamma-1) A_{t-1}(i)+u_{t}(i)_{\eta}^{\beta}\left(\bar{A}_{t}(i)-A_{t-1}(i)\right)\right]^{\sigma}
$$

Donde los parámetros $\gamma, \eta$ corresponden a las habilidades innovadoras e imitadoras adoptadas por las empresas en cada sector $i$, satisfaciendo las siguientes desigualdades: $\gamma>1$ (un paso adelante) y $\eta \leq 1$ (un paso atrás). El parámetro $\sigma$ refleja la elasticidad del componente estratégico, que por simplicidad se asume como $\sigma=1$. La frontera tecnológica crece a una tasa constante $\bar{g}, \bar{A}_{t}(i)=(1+\bar{g}) \bar{A}_{t-1}(i)$, y el estado tecnológico de cada sector está limitado al nivel de la frontera, por lo tanto, $\bar{A}_{t}(i) \leq \bar{A}_{t}(i)$. Los parámetros $\Phi, \beta$ representan los coeficientes de elasticidad de cada factor de producción. 


\section{Decisión del monopolista y condiciones de equilibrio}

El empresario monopolista decide invertir en la contratación de mano de obra calificada y no calificada, de acuerdo con su posición y con la mejor tasa de rendimiento de la inversión. Si su posición está relativamente lejos de la frontera tecnológica, la asignación de recursos a la contratación de mano de obra no calificada puede generar una mejora superior en relación con la contratación de mano de obra calificada, es decir, $\Delta u_{t}(i)^{\beta} \eta\left(\bar{A}_{t}(i)-A_{t-1}(i)>\Delta s_{t}(i)^{\Phi}(\gamma-1) A_{t-1}(i)\right.$.

La decisión del empresario monopolista depende de un problema de optimización entre los beneficios esperados y el costo de la inversión, en este caso representado por el gasto total en la contratación de mano de obra. Así, la función de utilidad del monopolista puede expresarse de la siguiente manera:

$$
U_{t}(i)=E(c)-W_{t}
$$

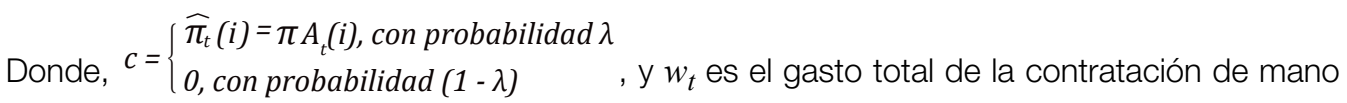
de obra calificada y no calificada. Los salarios de la fuerza de trabajo se denotan mediante $w^{u} A_{t-1}(i)$ (no calificada) y $w^{s} A_{t-1}(i)$ (calificada) ${ }^{2}$. Por definición, $w^{i} . \therefore i \in(s, u)$ corresponde a la tasa salarial. En consecuencia:

$$
W_{t}=w^{u} A_{t-1}(i) u_{t}(i)+w^{s} A_{t-1}(i) s_{t}(i)=\left(w^{u} u_{t}(i)+w^{s} s_{t}(i)\right) A_{t-1}(i)
$$

El problema de maximización de la utilidad del empresario se define como:

$$
\max _{u_{t}(i), s_{t}(i)} \max _{u_{t}(i), s_{t}(i)}\left\{\lambda \pi A_{t}-\left(w^{u} u_{t}(i)+w^{s} S_{t}(i)\right) A_{t-1}(i)\right\}
$$

La condición de primer orden con respecto a los argumentos $u_{t}(i), s_{t}(i)$ genera las siguientes ecuaciones de equilibrio:

$$
\begin{gathered}
\frac{\partial U}{\partial s_{t}(i)}=\lambda \pi \Phi_{t}(i)^{\Phi-1}(\gamma-1) A_{t-1}(i)-w^{s} A_{t-1}(i) \equiv 0 \\
\frac{\partial U}{\partial u_{t}(i)}=\lambda \pi \beta u_{t}(i)^{\beta-1} \eta\left(\bar{A}_{t-1}(i)-A_{t-1}(i)\right)-w^{u} A_{t-1}(i) \equiv 0
\end{gathered}
$$

Al reorganizar las ecuaciones $U .1$ y U.2, se obtienen las funciones de demanda del empresario monopolista en relación con la mano de obra calificada y no calificada. Estas funciones son estrictamente negativas con respecto a la tasa salarial y positivas con respecto a los demás argumentos de la función. A continuación, se detallan las funciones de la demanda:

$$
\begin{gathered}
\widehat{S}_{t}(i)=\left(\frac{\lambda \pi \Phi(\gamma-1)}{w^{s}}\right)^{\frac{1}{1-\phi}} \\
\widehat{u}_{t}(i)=\left(\frac{\lambda \pi \beta \eta\left(d_{t-1}(i)-1\right)}{w^{u}}\right)^{\frac{1}{1-\beta}} \therefore d_{t-1}=\frac{\bar{A}_{t-1}(i)}{A_{t-1}(i)}=\left(a_{t-1}\right)^{-1}
\end{gathered}
$$

Donde, " $a_{t-1}$ " representa el indicador de proximidad a la frontera y su inversa, la medida de distancia de la frontera.

Con el mercado de trabajo competitivo y en equilibrio, la demanda de fuerza de trabajo se iguala a la oferta, esta última dada de manera exógena $\widehat{u}_{t}(i)=\widetilde{U} ; \widehat{s}_{t}(i)=\widetilde{S}$. La interpretación de la ecuación E.1 muestra que la demanda de mano de obra calificada depende positivamente de la probabilidad de éxito $\lambda$ de la empresa innovadora, del parámetro de equilibrio de los beneficios $\pi$ y del tamaño incremental de la estrategia de innovación $\gamma-1$.

2 Los salarios son proporcionales a la productividad debido a que los avances tecnológicos aumentan los rendimientos de la mano de obra dedicada a sus actividades. En este sentido también, $w^{s}>>w^{u}$, señalando que la prima de calificación se vuelve mayor para la mano de obra más calificada. Véanse más detalles en Vandenbussche, Aghion y Meghir (2006) y Aghion y otros (2009). 
La segunda ecuación E.2 revela que la demanda de mano de obra no calificada del empresario es una función positiva de la distancia tecnológica $d_{t-1}(i)$ del sector $i$. En este sentido, el bajo nivel de aprendizaje de las empresas revela intereses a corto plazo dirigidos a estrategias de imitación. El elevado atraso de las empresas hace que los empresarios asignen una fracción de trabajadores no calificados a las actividades menos intensivas en innovación. A medida que el sector se acerque al nivel de la frontera, el empresario monopolista demandará cada vez más fuerza de trabajo dedicada a actividades de investigación y desarrollo (l+D).

Se destacan, además, importantes consideraciones sobre las funciones de demanda: no es el crecimiento aislado el que condiciona la demanda de factores del empresario, sino el crecimiento acompañado de la reducción de la brecha tecnológica entre las economías. A medida que la economía crece y reduce las diferencias, los cambios estructurales "condicionan" la demanda empresarial, que repercute nuevamente en el crecimiento y da lugar a nuevas demandas ${ }^{3}$.

\section{3. "Prima" de calificación}

De acuerdo con Acemoglu (1998), la "prima" de calificación representa las distorsiones salariales entre la mano de obra calificada y no calificada que inducen costos de oportunidad entre los factores de producción. De ese modo, una "prima" baja supone altos costos de oportunidad en la mano de obra calificada, que pueden explicarse por la baja remuneración vinculada a una demanda insuficiente, derivada del bajo desarrollo predominante en la economía. Al igual que en la "teoría de la empresa", el empresario busca un nivel de factor cuyo costo marginal se equipare a su valor marginal. La definición de "valor marginal" está asociada al concepto de productividad marginal del factor, que se vuelve proporcional al nivel de tecnología de la empresa. Los mayores niveles de productividad permiten incrementar el rendimiento del factor de producción, aumentando su "prima" de calificación.

En el presente estudio, la función "prima de calificación" puede expresarse mediante la relación entre los gastos de equilibrio de cada factor de producción:

$$
w_{t}(i) \equiv \frac{w^{s} A_{t-1}(i) \hat{s}_{t}(i)}{w^{u} A_{t-1}(i) \hat{u}_{t}(i)}=(\lambda \pi)^{\frac{\varphi-\beta}{(1-\varphi)(1-\beta)}} \cdot\left[\frac{(\varphi(\gamma-1))^{\frac{1}{1-\varphi}}}{\left(\beta \eta\left(d_{t-1}(i)-1\right)\right)^{\frac{1}{1-\beta}}}\right] \cdot \frac{\left(w^{u}\right)^{\frac{1}{1-\beta}}}{\left(w^{s}\right)^{\frac{1}{1-\varphi}}}
$$

De acuerdo con la ecuación W.1, la "prima de calificación" se vuelve inversamente determinada por la distancia con respecto a la frontera. Un bajo nivel de productividad en el sector supone una menor demanda de mano de obra calificada, de manera que se reduce el rendimiento de este factor y disminuye la "prima de calificación". Un incremento de las actividades de innovación $\gamma$ aumenta la "prima de calificación", de la misma forma que un incremento de las estrategias de imitación $\eta$ demanda un factor menos calificado y reduce la "prima de calificación".

En este caso, las mayores diferencias salariales suponen costos superiores para el factor más calificado, reduciendo su contribución al crecimiento de la productividad. Por el contrario, los patrones de productividad próximos a la frontera suponen un mayor rendimiento del factor más responsable de la "aproximación" de la empresa, aumentando la "prima de calificación". Por medio de este resultado, las fluctuaciones de proximidad con respecto a la frontera conllevan oscilaciones en la "prima de calificación".

\footnotetext{
3 Conforme al "enigma" de Dosi, Fagiolo y Roventini (2010), no solo el crecimiento conduce a los cambios tecnológicos a largo plazo, sino también los factores de demanda. ¿Está el crecimiento a largo plazo impulsado solamente por cambios en los "fundamentos" tecnológicos? ¿O pueden las variaciones en la demanda agregada influir en la dinámica futura? Y, en última instancia, ¿es posible identificar múltiples caminos de crecimiento cuya selección depende de la demanda y las condiciones institucionales? (Dosi, Fagiolo y Roventini, 2010, pág. 1.748).
} 


\section{Crecimiento y demanda de factor de producción}

A partir de las funciones de demanda E.1 y E.2, la tasa de progreso tecnológico puede reformularse de la siguiente manera:

$$
\begin{aligned}
& A_{t}(i)=A_{t-1}(i)+s_{t}(i)^{\phi}(\gamma-1) A_{t-1}(i)+u_{t}(i)^{\beta} \eta\left(\bar{A}_{t}(i)-A_{t-1}(i)\right) \sigma=1 \\
& g_{t}(i)=\frac{A_{t}(i)-A_{t-1}(i)}{A_{t-1}(i)}=\hat{s}_{t}(i)^{\Phi}(\gamma-1)+\hat{u}_{t}(i)^{\beta} \eta\left(d_{t-1}(i)-1\right)
\end{aligned}
$$

La tasa de progreso técnico es una función de las curvas de demanda, que establecen las condiciones de equilibrio de los parámetros de imitación, innovación y de la distancia con respecto a la frontera. Al sustituir E.1 y E.2 en G.1, se obtiene:

$$
g_{t}(i)=(\gamma-1)^{\frac{1}{1-\phi}}\left(\frac{\lambda \pi \Phi}{w^{S}}\right)^{\frac{\Phi}{1-\phi}}+\eta^{\frac{1}{1-\beta}}\left(\frac{\lambda \pi \beta}{w^{u}}\right)^{\frac{\beta}{1-\beta}}\left(d_{t-1}(i)-1\right)^{\frac{1}{1-\beta}}
$$

La ecuación G.2 presenta una importante conclusión del estudio:

- Proposición 1: Los sectores más alejados de la frontera tecnológica tienen una demanda de recursos diferenciada con respecto a los sectores más avanzados. En las economías más alejadas de la frontera existe un "déficit" en la demanda de factor-calificado que reduce la "prima de calificación" y su contribución al crecimiento de la productividad. Es decir, $d_{t-1}(i) \geq 1 \Rightarrow \hat{u}_{t}(i) \geq 0$.

La proposición 1 demuestra que la distancia con respecto a la frontera afecta directamente el crecimiento de la productividad, que está condicionado por la demanda de cada factor de producción. Esta conclusión puede presentarse mediante la derivada parcial de segundo orden con la función de crecimiento.

- Prueba: al reescribir la ecuación G.2, se obtiene:

$$
\frac{\partial^{2} g_{t}(i)}{\partial d_{t-1}(i) \partial \eta}=\left(\frac{\beta}{1-\beta}\right)^{2}\left(\frac{\lambda \pi \beta \eta\left(d_{t-1}(i)-1\right.}{w^{u}}\right)^{\frac{\beta}{1-\beta}} \geq 0 \Rightarrow d_{t-1}(i) \geq 1
$$

La ecuación G.3 muestra que la tasa de variación del progreso técnico depende positivamente de la distancia con respecto a la frontera. De existir un posible atraso $d_{t-1}(i)>1$, el aprendizaje por medio de prácticas intensivas de imitación y demanda de factor no calificado puede proporcionar oportunidades tecnológicas viables que refuercen el crecimiento a corto plazo. Por lo tanto, una "prima de calificación" baja es inducida por un déficit de demanda de factor calificado, que limita su contribución al crecimiento de la productividad.

\section{Metodología empírica}

\section{Definición de la muestra y operacionalización de las variables}

Los datos utilizados en este estudio se tomaron del Atlas de Desarrollo Humano en Brasil (PNUD/ IPEA/FJP, 2013), que incluye más de 200 indicadores de demografía, educación, ingresos, trabajo, vivienda y vulnerabilidad de los municipios brasileños a partir de los censos demográficos de 1991, 2000 y 2010 (IBGE, s/fa). 
Para analizar la información más reciente, se optó por delimitar el total de 5.565 municipios conforme al año 2010. Además, algunas variables importantes utilizadas en el estudio -como el ingreso medio de los ocupados - están disponibles exclusivamente en esta base de datos solo para $2010^{4}$. Con el fin de utilizar una muestra con el mayor número posible de datos y sin otras pérdidas de generalidad, el uso de datos de corte transversal no supuso graves limitaciones a la muestra, visto que los 5.565 municipios seleccionados son suficientes para una inferencia adecuada al modelo.

Las variables utilizadas en la investigación se describen en el cuadro 1.

Cuadro 1

Descripción de las variables utilizadas en el estudio

\begin{tabular}{ll}
\hline Sigla & Definición \\
\hline RDPC & Ingreso per cápita \\
\hline RENOCUP & Ingreso medio de los ocupados de 18 años o más \\
\hline$P_{\text {SUPER }}$ & Porcentaje de los ocupados con educación superior completa de 18 años o más \\
\hline PRENTRAB & Porcentaje del ingreso proveniente del trabajo \\
\hline$T_{\text {FBSUPER }}$ & Tasa bruta de asistencia, educación superior \\
\hline$P_{\text {MED }}$ & Porcentaje de los ocupados con educación secundaria completa de 18 años o más \\
\hline TRABCC & Porcentaje de empleados con contrato de trabajo de 18 años o más \\
\hline$P_{\text {TRANSF }}$ & Porcentaje de los ocupados en la industria de transformación de 18 años o más \\
\hline REN & Porcentaje de los ocupados sin ingresos de 18 años o más \\
\hline$E_{\text {ANOSESTUDO }}$ & Expectativa de años de estudio a los 18 años de edad \\
\hline$T_{\text {MED18M }}$ & Porcentaje de la población de 18 a 20 años con educación secundaria completa \\
\hline$T_{\text {FBMED }}$ & Tasa bruta de asistencia, educación secundaria \\
\hline$T_{\text {SUPER25M }}$ & Porcentaje de la población de 25 años o más con educación superior completa \\
\hline
\end{tabular}

Fuente: Programa de las Naciones Unidas para el Desarrollo (PNUD)/Instituto de Investigación Económica Aplicada (IPEA)/Fundación João Pinheiro (FJP), "Atlas de Desarrollo Humano en Brasil", 2013 [base de datos en línea] http://www.atlasbrasil.org. br/2013/es/consulta/.

En la próxima sección se describe el modelo de regresión lineal que busca analizar las relaciones entre las variables conforme a la descripción del modelo teórico.

\section{Modelo de regresión lineal}

El conjunto de ecuaciones que se han de estimar consiste en:

Reg.1

$$
\begin{aligned}
\log \left(R D P C_{i j}\right) & =\alpha+\beta_{1} \cdot P_{\text {SUPER }_{i j}}+\beta_{2} \cdot\left[P_{\text {SUPER }_{i j}} * w_{\text {relat }_{i j}}\right]+\beta_{3} \cdot P_{\text {TRANSF }_{i j}}+\beta_{4} \cdot R E N_{o_{i j}} \\
& +\beta_{5} \cdot E_{\text {ANOSESTUDO }_{i j}}+\beta_{6} \cdot T_{\text {MED18M }_{i j}}+\beta_{7} \cdot T_{\text {FBMED }_{i j}}+\beta_{8} \cdot T_{\text {SUPER } 25 M_{i j}} \\
& +\delta_{j} \cdot \varepsilon_{i j}
\end{aligned}
$$

\footnotetext{
4 Si bien lo más destacado del corte de datos para 2010 se basa en el Atlas de Desarrollo Humano en Brasil (PNUD/IPEA/FJP, 2013), cabe subrayar que otras bases de datos importantes pueden orientar la continuidad de esta investigación: la Pesquisa Nacional por Amostra de Domicílios (PNAD) (IBGE, s/fb) y los microdatos del Censo Demográfico 2010 (IBGE, s/fa). Estas bases de datos incluyen un nivel de información más detallado que, en principio, se correlaciona con los datos presentados en el Atlas.
} 
Reg.2

$$
\begin{aligned}
& P_{\text {SUPER }_{i j}}=\alpha^{\prime}+\beta_{1}^{\prime} \text { PRENTRAB }_{i j}+\beta_{2}^{\prime} \cdot T_{\text {FBSUPER }_{i j}}+\beta_{3}^{\prime} \cdot P_{M E D_{i j}}+\beta_{4}^{\prime} \cdot \text { TRABCC }_{i j} \\
& +\gamma \cdot x+\delta_{j}^{\prime}+\varepsilon_{i j}^{\prime}
\end{aligned}
$$

Reg.3

$$
\begin{aligned}
P_{\text {SUPER }_{i j}}{ }^{*} w_{\text {relat }_{i j}} & =\alpha^{\prime \prime}+\beta^{\prime \prime}{ }_{1} \operatorname{PRENTRAB}_{i j}+\beta^{\prime \prime}{ }_{2} \cdot T_{\text {FBSUPER }}+\beta^{\prime \prime}{ }_{3} \cdot P_{M E D_{i j}} \\
& +\beta^{\prime \prime}{ }_{4} \cdot \operatorname{TRABCC} C_{i j}+\theta \cdot x+\delta^{\prime \prime}{ }_{j}+\varepsilon^{\prime \prime}{ }_{i j}
\end{aligned}
$$

La ecuación Reg.1 constituye la ecuación principal del estudio. La ecuación analiza la contribución de la mano de obra calificada asignada a las actividades productivas, en la medida en que las oscilaciones en la remuneración relativa entre los municipios captan el costo de oportunidad entre los factores de producción.

Esta medida relativa de la remuneración está representada por la variable " $w$ relat" que corresponde a la relación entre el "ingreso medio de los ocupados" (RENOCUP) del municipio y el valor máximo observado en la unidad federativa a la que pertenece. Así, las distorsiones regionales no influyen en el cálculo de la medida relativa, de manera que la variable asume valores entre $0 \leq w_{\text {relat }} \leq 1$ para cada estado. Los valores cercanos a 1 representan municipios que se aproximan al mayor valor relativo a la remuneración media dentro de la unidad federativa, indicando ganancias superiores en la muestra regional y, por lo tanto, costos de oportunidad relativamente menores del factor-trabajo y viceversa. Esta medida relativa capta indirectamente el grado de proximidad con respecto a la frontera, conforme a la ecuación W.1. El vector $X$ agregado en las ecuaciones Reg.2 y Reg.3 representa los demás regresores de la ecuación Reg.1.

Los vectores $\delta_{j}, \delta_{j}^{\prime}, \delta^{\prime \prime}{ }_{j}$ representan el conjunto de efectos fijos relativos a las características particulares de cada unidad federativa. Esas características, debidamente incluidas en el modelo, captan diferentes patrones de desarrollo, políticas, demografía y otros aspectos que influyen en las relaciones entre las variables. En este caso, su exclusión implicaría un grave error de especificación en el modelo, que daría lugar a incoherencias en las estimaciones de los parámetros (Greene, 1999).

Las variables $\varepsilon_{i j}, \varepsilon_{i j}^{\prime}, \varepsilon^{\prime \prime}{ }_{i j}$ representan la medida de error o "ignorancia" y, por lo tanto, captan los demás factores irrelevantes para el modelo.

\section{Método de estimación}

Las ecuaciones Reg.1-3 presentan una relación de endogeneidad que es necesario abordar adecuadamente en el proceso de estimación de los parámetros. Descuidarla implica graves errores de especificación y método, que causan sesgos e incoherencias en la técnica. Uno de los enfoques más populares para solucionar este problema metodológico es el estimador de variables instrumentales (Galvao y Montes-Rojas, 2015).

A su vez, el método debe cumplir las siguientes condiciones de validez a fin de garantizar estimaciones sin sesgos ni incoherencias de los parámetros (Hsiao y Zhang, 2015): los instrumentos (variables exógenas al modelo) deben ser i) válidos y, por lo tanto, no correlacionados con la perturbación estocástica; y ii) relevantes, de manera que capten información importante sobre los regresores endógenos.

Para poner a prueba las hipótesis descritas, se implementaron dos importantes estadísticas de prueba con el fin de evaluar la idoneidad de los instrumentos seleccionados. Estas estadísticas se describen en el cuadro 2. 
Cuadro 2

Resumen de las estadísticas de prueba del modelo

\begin{tabular}{lll}
\hline Pruebas & & \multicolumn{2}{c}{ Resumen de las estadísticas } \\
\hline J-Hansen (Hayashi, 2000) & Hipótesis nula $\left(\mathrm{H}_{0}\right)$ & Hipótesis alternativa $\left(\mathrm{H}_{\mathrm{a}}\right)$ \\
\cline { 2 - 3 } & $\begin{array}{l}\text { Instrumentos válidos y no correlacionados } \\
\text { con el error }\end{array}$ & Instrumentos no válidos y correlacionados con el error \\
\hline Kleibergen y Paap (2006) & Hipótesis nula $\left(\mathrm{H}_{0}\right)$ & Hipótesis alternativa $\left(\mathrm{H}_{\mathrm{a}}\right)$ \\
\cline { 2 - 3 } & $\begin{array}{l}\text { Instrumentos no relevantes y débilmente o no } \\
\text { correlacionados con los regresores endógenos }\end{array}$ & $\begin{array}{l}\text { Instrumentos relevantes y fuertemente correlacionados } \\
\text { con los regresores endógenos }\end{array}$ \\
\hline
\end{tabular}

Fuente: Elaboración propia.

Si bien los resultados de las estadísticas indican una selección de instrumentos adecuada, la técnica de variables instrumentales está sujeta a la pérdida de eficiencia de las estimaciones cuando hay problemas de heterogeneidad en el modelo estimado. Esta violación de la hipótesis estadística induce un sesgo en las estimaciones del cuadro 2, de manera que su corrección se vuelve necesaria para llegar a una conclusión adecuada sobre los instrumentos y el resultado del modelo (Hayashi, 2000; Baum, Schaffer y Stillman, 2003).

Para verificar la presencia de heterocedasticidad, se aplicó al modelo la prueba de Pagan y Hall (1983) adaptada a la técnica de variables instrumentales (Baum, Schaffer y Stillman, 2003). Una vez diagnosticada su presencia, se implementó la técnica de corrección por medio de agrupación residual, tomando las unidades federativas como patrón de grupo (clúster). Este procedimiento conduce a estimaciones eficientes, permitiendo las subsiguientes pruebas de adecuación de los instrumentos más fiables y consistentes.

Por último, para garantizar alternativas de estimación y una mayor precisión, se aplicó al modelo el método generalizado de momentos, en referencia a las contribuciones de Hansen (1982). En presencia de heterocedasticidad, el método generalizado de momentos presenta ganancias de eficiencia en sus estimaciones en relación con los estimadores de variables instrumentales tradicionales (Baum, Schaffer y Stillman, 2003). Estos resultados también se evidenciaron recientemente en Cameron y Trivedi (2005) y Hsiao y Zhang (2015).

En el cuadro 3 se describen las variables utilizadas en el modelo.

Cuadro 3

Descripción de las variables en el método de estimación

\begin{tabular}{lll}
\hline Variables instrumentadas & Instrumentos incluidos & Instrumentos excluidos \\
\hline$P_{\text {SUPER }}$ & $P_{\text {TRANSF }}$ & PRENTRAB \\
\hline$\left[P_{\text {SUPER }}{ }^{*} W_{\text {relat }}\right]$ & REN & $T_{\text {FBSUPER }}$ \\
\hline & $E_{\text {ANOSESTUDO }}$ & TRABCC \\
\hline & $T_{\text {MED18M }}$ & $P_{\text {MED }}$ \\
\hline & $T_{\text {FBMED }}$ & \\
\hline & $T_{\text {SUPER25M }}$ & \\
\hline
\end{tabular}

Fuente: Elaboración propia.

\section{Selección de los instrumentos}

Para captar los factores condicionantes de la reserva de capital humano y las diferencias en el mercado de trabajo, en algunos estudios recientes -en especial, Philippon y Reshef (2012) y Gennaioli y otros (2013) - se ha señalado que las diferencias en capital humano constituyen importantes fuentes de desigualdad regional y persisten a lo largo del tiempo en varias regiones del mundo. Como todo capital, debe acumularse por medio del esfuerzo de las personas, que proyectan un rendimiento a partir de las competencias adquiridas (Lucas, 2009). En este caso, el ingreso proveniente del trabajo sirve como recurso disponible para financiar la ampliación de las competencias, que se traduce en nuevas fuentes de asimetría en la distribución de los salarios. 
Gennaioli y otros (2013) determinaron que los salarios son relativamente más altos en las regiones más productivas y desarrolladas y que esta diferencia intensifica el efecto migratorio desde las regiones menos desarrolladas. La mano de obra calificada que vive en las regiones menos productivas es incentivada a migrar a causa del elevado costo de oportunidad entre los factores. Así, los trabajadores más calificados tienen un mayor incentivo para pagar el costo de la migración porque los beneficios recibidos son notablemente mayores. Esto amplía aún más la reserva de calificación, aumentando la demanda de nuevos cursos de capacitación a raíz de la propia dinámica local. El aumento de la demanda de nuevas formas de calificación incrementa las inscripciones en los cursos de capacitación, que tienden a ser relativamente mayores en las regiones más desarrolladas. Esto promueve una reasignación de las competencias en el mercado de trabajo, en la que se da más peso a la mano de obra altamente calificada que a la mano de obra poco calificada (Schiopu, 2015).

Recientemente, Bastgen y Holzner (2017) evidenciaron una importante relación entre el desarrollo, la innovación y la protección en el mercado de trabajo. Según los autores, el empleo formal y su protección por la legislación hacen que las empresas mantengan a los trabajadores empleados incluso en períodos de baja productividad. El hecho de mantener a los profesionales empleados en épocas de baja productividad induce a las empresas a aumentar sus iniciativas en materia de innovación para compensar este proceso. Los avances tecnológicos compensan la disminución de la productividad y estimulan aún más la formación de mano de obra calificada. Este proceso modifica la actividad económica local, generando más desarrollo y reforzando la relación entre la protección en el mercado de trabajo y la innovación. Así, una legislación que protege el mercado laboral estimula la innovación y la entrada de nuevas empresas, de manera que muchos países con una estricta legislación laboral tienden a estimular y a especializarse en la mejora de productos y procesos existentes (Acharya, Baghai y Subramanian, 2014; Koeniger, 2005).

\section{Análisis de los resultados}

\section{Análisis descriptivo de la muestra}

En el cuadro 4 se presentan las principales estadísticas descriptivas de la muestra de datos.

Cuadro 4

Estadísticas descriptivas de la muestra

\begin{tabular}{lrrrr}
\hline Variable & Media & Desviación estándar & Mínimo & Máximo \\
\hline RDPC & 493,61 & 243,27 & 96,25 & 2043,74 \\
\hline RENOCUP & 780,11 & 341,68 & 136,42 & 3177,26 \\
\hline$P_{\text {SUPER }}$ & 7,04 & 3,61 & 0,32 & 37,53 \\
\hline PRENTRAB & 68,48 & 10,80 & 27,43 & 95,24 \\
\hline$T_{\text {FBSUPER }}$ & 19,10 & 10,38 & 0,96 & 76,78 \\
\hline$P_{\text {MED }}$ & 30,42 & 9,84 & 4,16 & 73,65 \\
\hline TRABCC & 30,25 & 18,05 & 0,90 & 83,21 \\
\hline$P_{\text {TRANSF }}$ & 9,61 & 8,92 & 0,00 & 65,11 \\
\hline REN & 13,42 & 11,98 & 0,00 & 73,93 \\
\hline EANOSESTUDO $_{\text {AnA }}$ & 9,46 & 1,10 & 4,34 & 12,83 \\
\hline$T_{\text {MED18M }}$ & 24,75 & 8,68 & 3,04 & 66,23 \\
\hline$T_{\text {FBMED }}$ & 65,85 & 14,97 & 0,00 & 168,64 \\
\hline$T_{\text {SUPER25M }}$ & 5,50 & 3,26 & 0,28 & 33,68 \\
\hline
\end{tabular}

Fuente: Elaboración propia. 
El ingreso per cápita de los municipios fue de 493,61 reales, entre un valor mínimo de 96,25 reales y uno máximo de $2.043,74$ reales. Considerando el ingreso de los ocupados, el valor medio aumenta a 780,11 reales (una variación correspondiente al 58,04\%), con un mínimo de 136,42 reales y un máximo de $3.177,26$ reales.

La proporción media de ocupados con educación superior completa en los municipios fue de alrededor del 7\%, con un mínimo del 0,32\% y un máximo del 37,53\%. Con respecto al ingreso proveniente del trabajo, el mayor valor observado asciende al 95,24\% (en este municipio, el 95,24\% del ingreso total proviene del trabajo), mientras la media corresponde al 68,48\%.

La proporción de ocupados con educación secundaria completa resultó ser bastante más alta que la de aquellos con educación superior, con un 30,42\% de los ocupados. La menor proporción registrada fue de aproximadamente el 4\%, mientras el máximo valor observado superó el $70 \%$.

El porcentaje de trabajadores con contrato de trabajo capta parcialmente el nivel de formalidad en la economía local. Al definir esta parte de la formalidad en función de los trabajadores con contrato de trabajo, en esta contabilidad no se tiene en cuenta al sector público ${ }^{5}$. De acuerdo con algunas investigaciones recientes, en especial Mattos (2011 y 2015), el empleo público representa alrededor del $10,7 \%$ de las personas ocupadas en el Brasil (en el sentido más amplio, que abarca la administración directa, indirecta y estatal de todo tipo). Así, se considera que esta baja proporción no supondrá grandes pérdidas de calidad asintótica para el modelo, ya que se aplicarán pruebas a los instrumentos para su debida validación (Greene, 1999).

Los datos de los municipios muestran una baja proporción de trabajadores con contrato de trabajo, pues la media representa aproximadamente el 30,25\% de las personas ocupadas en el grupo etario de referencia. La proporción máxima corresponde al 83,21\%, de manera que solo el 16,79\% de los trabajadores ocupados no tendrían contrato de trabajo en ese municipio.

Otro dato importante relativo al mercado de trabajo consiste en la ocupación en la industria de transformación. El porcentaje medio de trabajadores ocupados en este sector fue de aproximadamente el 9,6\%, con un valor máximo en la muestra del $65,11 \%$.

Al analizar el porcentaje de trabajadores ocupados sin ingresos, la proporción media se situó alrededor del $13,42 \%$. Esta variable presentó un valor mínimo nulo y un valor máximo del $73,93 \%$. Esto indica que la desigualdad relativa a la falta de remuneración del trabajo todavía prevalece en algunos municipios de la muestra.

Una dimensión importante del desarrollo municipal es la expectativa de educación, que proyecta la expectativa de años de estudio para las generaciones futuras. La expectativa media fue de 9,46 años de estudio, con un valor máximo de 12,83.

La proporción de la población con educación secundaria completa alcanzó un valor medio bajo, del $24,75 \%$. El municipio con el mayor valor presentó una proporción de graduados de la educación secundaria del 66,23\%. La proporción de la población de 25 años o más con educación superior completa también alcanzó una media relativamente baja, del 5,5\%, y un valor máximo del 33,68\% (por debajo de las proporciones relativas a la fuerza de trabajo ocupada).

En el cuadro 5 se presenta la matriz de correlación entre las variables utilizadas en el modelo. La variable $\log (\mathrm{RDPC})$ presentó una correlación significativa con todas las variables seleccionadas (significativas al 1\%). La mayor correlación con log(RDPC) correspondió a la variable $\mathrm{REN}_{0}$ que representa el porcentaje de trabajadores ocupados sin ingresos $(\rho=-0,737)$, seguida de la variable proporción de personas con educación secundaria completa $(\rho=0,736)$.

5 Esta variable excluye a los militares del ejército, la marina, la aeronáutica, la policía militar y el cuerpo de bomberos, los empleados bajo el régimen jurídico de los funcionarios públicos, así como a los empleadores y los trabajadores por cuenta propia que eran contribuyentes de un instituto de previsión oficial, ya que estas categorías presentan una dinámica diferenciada en el mercado de trabajo. 


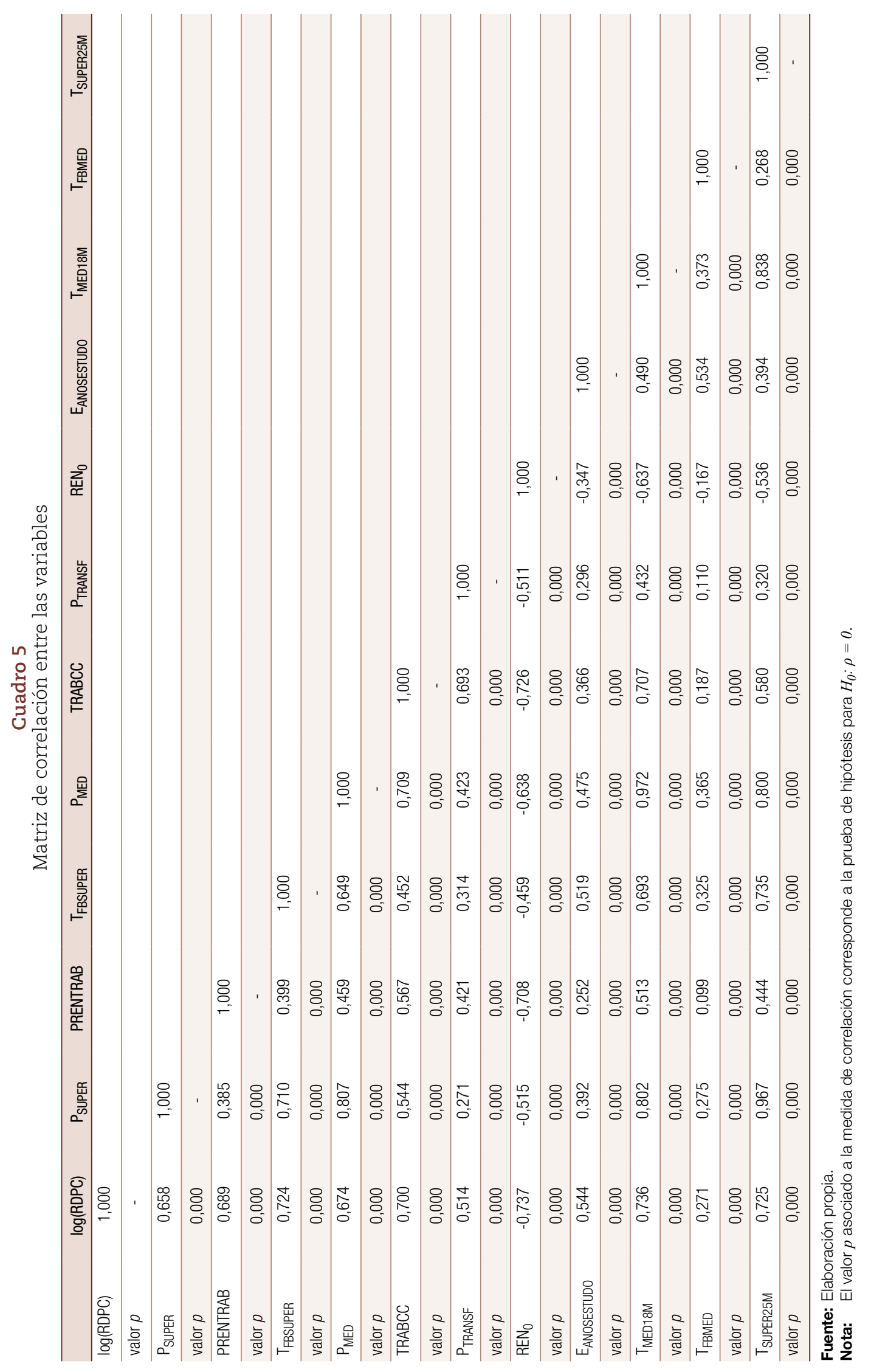


Solo una variable presentó un coeficiente de correlación bajo, la tasa bruta de asistencia de la educación secundaria $(\rho=0,271$, aunque significativa al nivel del 1\%). Todas las demás variables presentaron coeficientes de correlación superiores a $\rho=0,50$, mostrando correlaciones moderadas y altas.

\section{Análisis del modelo de regresión}

Los resultados del modelo estimado conforme a la técnica de mínimos cuadrados ordinarios, con la inclusión de los efectos fijos para cada unidad federativa, se detallan en el cuadro 6.

Cuadro 6

Resultados del modelo econométrico

\begin{tabular}{|c|c|c|}
\hline Variables & $\begin{array}{c}\text { (1) } \\
\text { Mínimos cuadrados ordinarios } \\
\text { Efectos fijos }\end{array}$ & $\begin{array}{c}\text { (2) } \\
\text { Mínimos cuadrados ordinarios } \\
\text { Efectos fijos }\end{array}$ \\
\hline \multirow[t]{2}{*}{$P_{\text {SUPER }}$} & $-0,0252^{\star \star}$ & $-0,0653^{\star \star \star}$ \\
\hline & $(0,00940)$ & $(0,00771)$ \\
\hline \multirow[t]{2}{*}{$\mathrm{P}_{\text {SUPER }}{ }^{*} \mathrm{~W}_{\text {relat }}$} & $0,115^{\star \star \star}$ & $0,0646^{\star \star \star}$ \\
\hline & $(0,0153)$ & $(0,0102)$ \\
\hline \multirow[t]{2}{*}{$P_{\text {TRANSF }}$} & & $0,00311^{\star \star \star}$ \\
\hline & & $(0,000495)$ \\
\hline \multirow[t]{2}{*}{$\mathrm{REN}_{0}$} & & $-0,00949^{* \star \star}$ \\
\hline & & $(0,00127)$ \\
\hline \multirow[t]{2}{*}{$\mathrm{E}_{\text {ANOSESTUDO }}$} & & $0,0316^{\star \star \star}$ \\
\hline & & $(0,00573)$ \\
\hline \multirow[t]{2}{*}{$\mathrm{T}_{\text {MED18M }}$} & & $0,00679^{\star \star \star}$ \\
\hline & & $(0,00141)$ \\
\hline \multirow[t]{2}{*}{$T_{\text {FBMED }}$} & & 0,000383 \\
\hline & & $(0,000407)$ \\
\hline \multirow[t]{2}{*}{$\mathrm{T}_{\text {SUPER25M }}$} & & $0,0603^{\star \star \star}$ \\
\hline & & $(0,00797)$ \\
\hline \multirow[t]{2}{*}{ Constante } & $5,477^{\star \star *}$ & $5,371^{\star \star \star}$ \\
\hline & $(0,0287)$ & $(0,0512)$ \\
\hline Efectos fijos & - & - \\
\hline - Estado & Sí & Sí \\
\hline Prueba de heterocedasticidad & - & - \\
\hline$-\chi^{2}$ & 734,65 & 98,44 \\
\hline - Valor $p$ & 0,0000 & 0,0000 \\
\hline $\mathrm{R}^{2}$ & 0,846 & 0,906 \\
\hline$R^{2}-A d j$ & 0,845 & 0,906 \\
\hline Estadística F & 1082,87 & 1573,29 \\
\hline Estadística $\mathrm{F}$ (valor $p$ ) & 0,0000 & 0,0000 \\
\hline
\end{tabular}

Fuente: Elaboración propia.

Nota: $\quad{ }^{* \star *} p<0,01,{ }^{\star \star} p<0,05,{ }^{*} p<0,1$.

La confirmación en las pruebas de heterocedasticidad supuso volver a calcular la matriz de covarianza de los parámetros siguiendo la técnica de agrupación residual tomando las unidades federativas como referencia en los grupos (clústeres).

Conforme a los resultados detallados en el cuadro, el parámetro vinculado a la variable "proporción de ocupados con educación superior completa" presentó un valor negativo en las dos columnas (significativo al nivel del 1\%). El parámetro representa una semielasticidad de la variable en el ingreso per cápita de los municipios. Así, sin tener en cuenta la influencia de las diferencias salariales en la demanda de mano de obra calificada, un aumento de una unidad en la proporción de ocupados contribuye a una reducción media del 2,6\% (columna (1)) y del 6,7\% (columna (2)) [(e $\beta-1) * 100]$. Sin embargo, este efecto medio no es coherente con la literatura presentada, lo que sugiere que el signo vinculado 
al parámetro estimado está influenciado por las diferencias salariales conforme al modelo presentado. En este caso, el parámetro asociado al efecto cruzado se presenta como positivo, lo que indica que el efecto final de la relación depende del factor de aproximación entre los mayores beneficios (ingreso máximo para cada unidad federativa).

Estos datos empíricos sugieren que el factor-diferenciación entre los ingresos señala la existencia de costos de oportunidad entre los factores. Así, en promedio, los municipios más desarrollados administran mayores beneficios, reduciendo los costos de oportunidad entre los factores (véase el gráfico 1).

Gráfico 1

Relación entre el ingreso medio de los ocupados y el ingreso per cápita

(En reales)

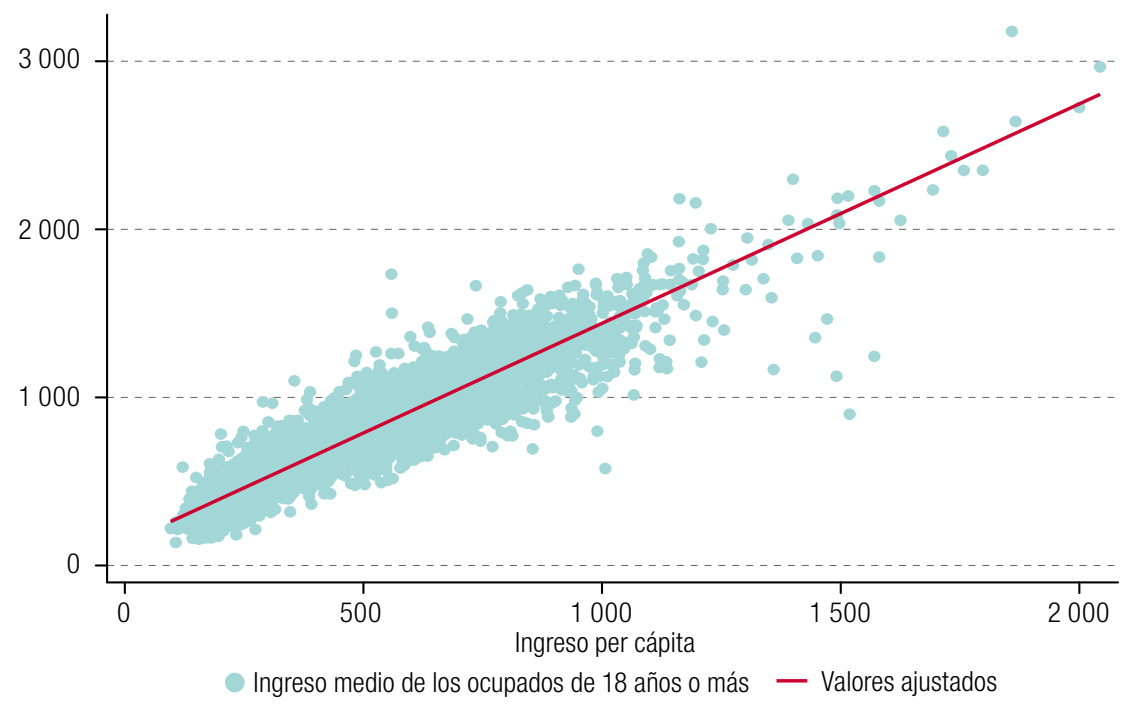

Fuente: Elaboración propia.

El parámetro correspondiente al porcentaje de los ocupados sin ingresos presentó el signo negativo esperado y fue significativo al nivel del $1 \%$. Así, en promedio, un aumento de una unidad en la proporción tiende a reducir el ingreso per cápita un 0,95\%. La expectativa de años de estudio presentó la relación positiva esperada. En promedio, un aumento adicional de la expectativa de años de estudio contribuye a aumentar el ingreso per cápita un 3,21\%.

Al comparar la proporción de la población con educación secundaria y superior completas, ambos parámetros presentaron los signos positivos esperados y significación estadística (nivel del 1\%). Sin embargo, un aumento en la proporción de la población con educación secundaria completa contribuye a un aumento de aproximadamente un 0,68\% en el ingreso per cápita, en comparación con un aumento del 6,22\% en el caso de un incremento en la proporción de graduados con educación superior.

Las pruebas de heterocedasticidad presentaron un rechazo de la hipótesis nula al nivel del $1 \%$ de varianza homocedástica. De esta forma, la matriz de varianza-covarianza volvió a calcularse para que las estimaciones de error estándar fueran coherentes (parámetros eficientes). El poder de explicación del modelo presentó un valor elevado en las columnas (1) y (2), además de medidas de R2-ajustado cercanas al poder de explicación. Los modelos presentaron significación global a partir de los valores elevados en las estadísticas $\mathrm{F}$ y los correspondientes valores $p$ (significativos al nivel del 1\%).

En el gráfico 2 se muestra que la aproximación de los ingresos al valor máximo en cada unidad federativa incrementa el rendimiento del capital humano altamente calificado en el ingreso per cápita de los municipios. 
Gráfico 2

Simulación a partir de los resultados del modelo estimado

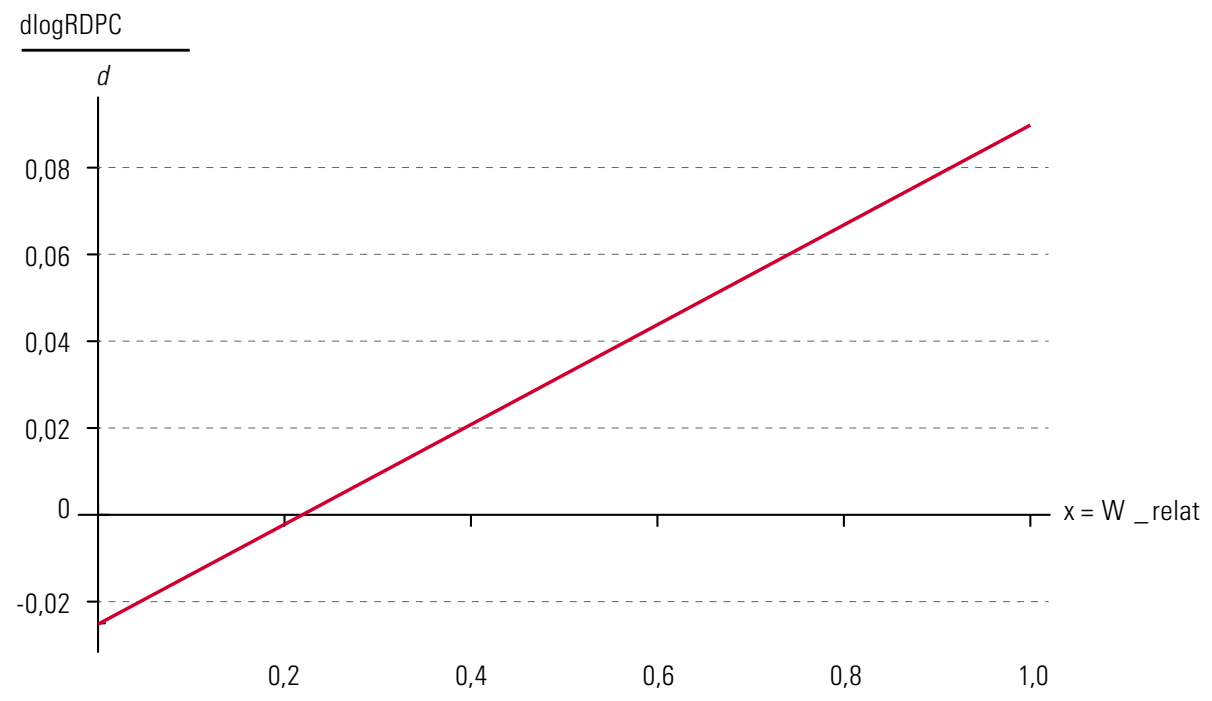

Fuente: Elaboración propia.

Nota: Simulación a partir de los resultados del cuadro 6, columna (1).

Como se muestra en el gráfico 2, el efecto final de la mano de obra altamente calificada está condicionado por las diferencias salariales. El gráfico revela que el efecto positivo se estima a partir de una diferencia de 0,22 o del 22\%. Así, en los municipios cuyo ingreso medio alcanza valores superiores al 22\% hasta el máximo observado en la unidad federativa, la expansión de la mano de obra con educación superior conduce a una relación positiva con el aumento del ingreso per cápita.

Este análisis indica que la relación entre el "capital humano" y sus canales de efecto en la generación de riqueza presentan resultados heterogéneos que, a veces, no se abordan debidamente en el análisis. Algunos factores relativos a los diferentes costos de oportunidad entre los factores pueden condicionar tales efectos, conduciendo a un sesgo en la relación entre las variables, que aumenta a medida que dichas diferencias regionales se vuelven más significativas.

En el cuadro 7 se presentan los resultados conforme a las técnicas de variables instrumentales y método generalizado de momentos.

En las columnas (1) y (3) se presentan los resultados del modelo simplificado, que indican el mismo entendimiento del cuadro 6 . Se observa un efecto negativo del parámetro asociado a la variable "proporción de ocupados con educación superior completa" (significativo al 1\% en las dos columnas). El parámetro asociado al efecto cruzado se presenta como positivo, indicando que el efecto final de la relación depende de las diferencias salariales que reflejan costos de oportunidades distintos entre los municipios (parámetros significativos al 1\%). Si bien las diferencias en los parámetros (variables instrumentales-método generalizado de momentos) presentan magnitudes relativamente pequeñas, las estadísticas $F$ presentan discrepancias mayores, indicando ganancias de eficiencia en el método generalizado de momentos.

Considerando las columnas (2) y (4), la inclusión de las demás variables influye en la estimación de los principales parámetros del modelo, de manera que su exclusión sobrestima las estimaciones. E aumento adicional en la "proporción de ocupados con educación superior completa" en el municipio con mayor ingreso medio de los ocupados, contribuye de media a un aumento del ingreso per cápita de los municipios de aproximadamente el 12,98\% (columna (2)) y el 15,84\% (columna (4)), en comparación con el $20,80 \%$ (columna (1)) y el $18,77 \%$ (columna (3)). Considerando este efecto conforme a los resultados del cuadro 6 , su magnitud representa un valor medio del 9,40\% para el mismo municipio. 


\section{Cuadro 7}

Resultados del modelo econométrico según las técnicas de variables instrumentales y método generalizado de momentos

\begin{tabular}{|c|c|c|c|c|}
\hline Variables & $\begin{array}{c}\text { (1) } \\
\text { Variables } \\
\text { instrumentales } \\
\text { Efectos fijos }\end{array}$ & $\begin{array}{c}\text { (2) } \\
\text { Variables } \\
\text { instrumentales } \\
\text { Efectos fijos }\end{array}$ & $\begin{array}{c}\text { (3) } \\
\text { Método generalizado } \\
\text { de momentos } \\
\text { Efectos fijos }\end{array}$ & $\begin{array}{c}\text { (4) } \\
\text { Método generalizado } \\
\text { de momentos } \\
\text { Efectos fijos }\end{array}$ \\
\hline \multirow[t]{2}{*}{$P_{\text {SUPER }}$} & $-0,177^{\star \star \star}$ & $-0,138^{\star \star *}$ & $-0,168^{\star \star *}$ & $-0,125^{\star \star \star}$ \\
\hline & $(0,0244)$ & $(0,0161)$ & $(0,0235)$ & $(0,0144)$ \\
\hline \multirow[t]{2}{*}{$P_{\text {SUPER }}{ }^{*} W_{\text {relat }}$} & $0,366^{\star \star \star}$ & $0,260^{\star \star \star}$ & $0,340^{\star \star \star}$ & $0,272^{\star \star \star}$ \\
\hline & $(0,0437)$ & $(0,0403)$ & $(0,0359)$ & $(0,0387)$ \\
\hline \multirow{2}{*}{$\mathrm{P}_{\text {TRANSF }}$} & & $0,00403^{\star \star \star}$ & & $0,00503^{\star \star \star}$ \\
\hline & & $(0,00130)$ & & $(0,000860)$ \\
\hline \multirow[t]{2}{*}{$\mathrm{REN}_{0}$} & & $-0,00613^{\star \star \star}$ & & $-0,00658^{* * *}$ \\
\hline & & $(0,00105)$ & & $(0,000852)$ \\
\hline \multirow[t]{2}{*}{$\mathrm{E}_{\text {ANOSESTUDO }}$} & & $0,0717^{\star \star \star}$ & & $0,0747^{\star \star \star}$ \\
\hline & & $(0,0121)$ & & $(0,0104)$ \\
\hline \multirow[t]{2}{*}{$\mathrm{T}_{\mathrm{MED18M}}$} & & $0,00528^{\star \star \star}$ & & $0,00539^{* \star \star}$ \\
\hline & & $(0,00170)$ & & $(0,00152)$ \\
\hline \multirow[t]{2}{*}{$\mathrm{T}_{\text {FBMED }}$} & & $9,81 e-05$ & & $-0,000242$ \\
\hline & & $(0,000439)$ & & $(0,000370)$ \\
\hline \multirow{2}{*}{$\mathrm{T}_{\text {SUPER25M }}$} & & $-0,00624$ & & $-0,0280$ \\
\hline & & $(0,0224)$ & & $(0,0186)$ \\
\hline \multirow[t]{2}{*}{ Constante } & $6,134^{\star \star \star}$ & $5,508^{\star \star \star}$ & $6,190^{\star \star \star}$ & $5,487^{\star \star \star}$ \\
\hline & $(0,118)$ & $(0,155)$ & $(0,0992)$ & $(0,133)$ \\
\hline Efectos fijos & - & - & - & - \\
\hline - Estado & Sí & Sí & Sí & Sí \\
\hline Prueba de heterocedasticidad & - & - & - & - \\
\hline$-\chi^{2}$ & 212,550 & 75,498 & 212,550 & 75,498 \\
\hline - Valor $p$ & 0,0000 & 0,0000 & 0,0000 & 0,0000 \\
\hline Estadística F & 52,97 & 89,57 & 82,30 & 104,3 \\
\hline Estadística $\mathrm{F}$ (valor $p$ ) & 0,0000 & 0,0000 & 0,0000 & 0,0000 \\
\hline Estadística LM Kleibergen-Paap $\left(\chi^{2}\right)$ & 14,02 & 16,87 & 14,02 & 16,87 \\
\hline Estadística LM Kleibergen-Paap (valor $p$ ) & 0,00288 & 0,000751 & 0,00288 & 0,000751 \\
\hline Estadística J de Hansen $\left(\chi^{2}\right)$ & 2,015 & 3,056 & 2,015 & 3,056 \\
\hline Estadística J de Hansen (valor $p$ ) & 0,365 & 0,217 & 0,365 & 0,217 \\
\hline
\end{tabular}

Fuente: Elaboración propia.

Nota: $\quad{ }^{* * \star} p<0,01,{ }^{* *} p<0,05,{ }^{*} p<0,1$.

La confirmación en las pruebas de heterocedasticidad supuso volver a calcular la matriz de covarianza de los parámetros siguiendo la técnica de agrupación residual tomando las unidades federativas como referencia en los grupos (clústeres).

El divisor entre los efectos positivos y negativos de la expansión de la mano de obra ocupada con educación superior en el ingreso per cápita $\left(\frac{\partial \log (R D P C)}{\partial P_{\text {SUPER }}}=\hat{\beta}_{1}+\hat{\beta}_{2} \cdot w_{\text {relat }} \geq 0\right)$ representa distintos resultados entre los modelos. Controlando la endogeneidad de los regresores, los valores relativos entre el ingreso medio de los ocupados por encima del 46\% (véase el cuadro 7) presentan una relación positiva entre las variables. Sin tener en cuenta la endogeneidad (véase el cuadro 6), el valor relativo representa una magnitud del $22 \%$, demostrando un sesgo inferior equivalente a casi el $50 \%$ hacia abajo con respecto al método de variables instrumentales y el método generalizado de momentos. Este resultado se presenta en el gráfico 3, en el que se simula la relación entre el ingreso per cápita y la proporción de ocupados con educación superior, según diferentes valores relativos de ingreso entre los municipios. 
Gráfico 3

Simulación a partir de los resultados del modelo estimado

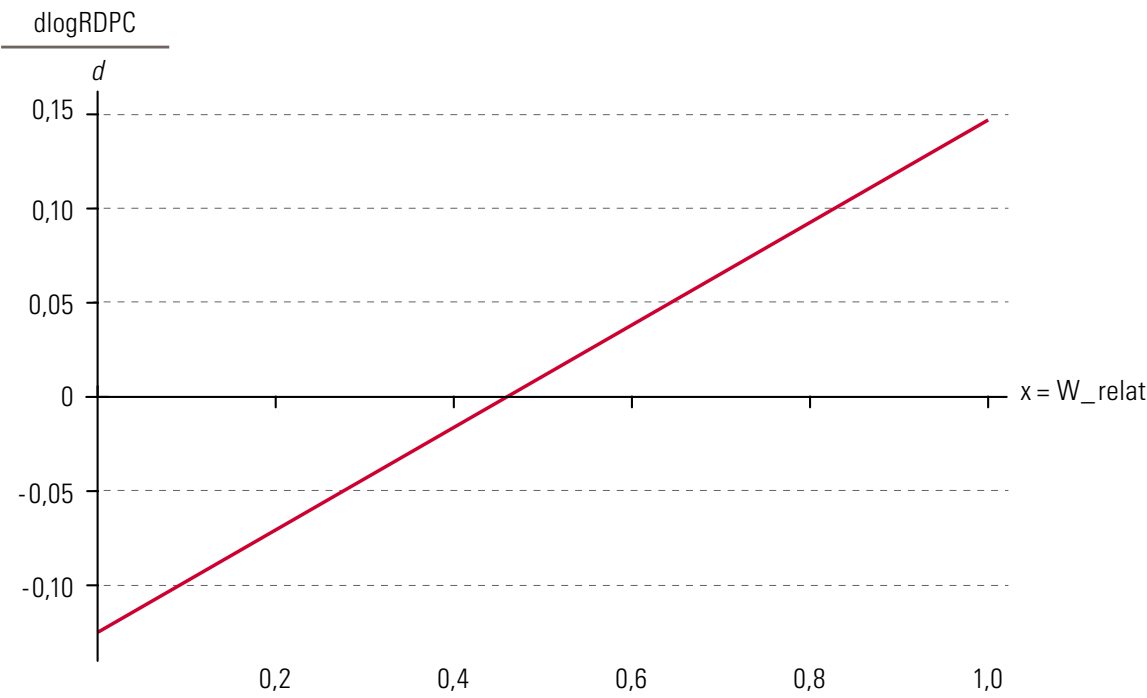

Fuente: Elaboración propia.

Nota: Simulación a partir de los resultados del cuadro 7, columna (4).

Es importante destacar que las variables "tasa bruta de asistencia a la educación secundaria" y "porcentaje de la población de 25 años o más con educación superior completa" presentaron signos negativos y falta de significación estadística, al contrario de los resultados del cuadro 6 . El impacto de la "expectativa de años de estudio" en la acumulación del ingreso per cápita prácticamente se duplicó al controlar los problemas relativos de endogeneidad en el modelo (0,0717 y 0,0747 en las columnas (2) y (4) del cuadro 7 , respectivamente, frente a 0,0316 en la columna (2) del cuadro 6), parámetros significativos al 1\%.

Las pruebas de heterocedasticidad revelaron signos de presencia significativa en todas las columnas (rechazo de la hipótesis nula al 1\%). Esto demuestra la necesidad del método generalizado de momentos para aumentar la eficiencia.

En todas las columnas del cuadro 7, las pruebas de validez y relevancia de los instrumentos mostraron que no hay signos significativos de correlación con la perturbación estocástica (valor $p$ elevado en las pruebas $\mathrm{J}$ de Hansen), además de una elevada correlación entre los instrumentos y los regresores endógenos.

\section{Discusión de los resultados}

Los resultados presentados indican que el aumento en la "proporción de ocupados con educación superior completa" tiende a contribuir a un mayor rendimiento en la acumulación del ingreso per cápita en los municipios cuyos costos de oportunidad del factor sean relativamente menores. En este caso, los municipios más desarrollados, con mayores niveles de ingreso per cápita e ingresos salariales, ofrecen mejores incentivos a una mano de obra más calificada. Esto demuestra que la contribución del capital humano al aumento de la riqueza presenta resultados heterogéneos, que están condicionados por las distintas realidades regionales. Otros estudios regionales han respaldado estos resultados, entre los que se destacan Aghion y otros (2009) y Zhang y Zhuang (2011).

En un estudio sobre la composición de las inversiones en educación en los Estados Unidos, Aghion y otros (2009) encontraron indicios de que las inversiones en educación superior estimulaban un mayor crecimiento en los estados con mayores niveles de desarrollo tecnológico y, por lo tanto, 
más cercanos a la frontera tecnológica. En los estados que se encuentran en la frontera tecnológica, un aumento de 1.000 dólares en las inversiones per cápita en investigación en la educación superior contribuye a un aumento medio de la tasa de crecimiento de dichos estados de aproximadamente 0,04 puntos porcentuales. El resultado final de las inversiones depende del coeficiente de proximidad con respecto a la frontera, de manera de generar resultados positivos o incluso negativos para los estados más alejados.

Zhang y Zhuang (2011) evaluaron los efectos de la composición del capital humano en el crecimiento de China, distinguiendo tres niveles: terciario, secundario y primario. Según los autores, el papel de la composición del capital humano en el crecimiento regional es relevante y está influenciado por las disparidades de desarrollo dentro del país. Los beneficios de la mano de obra con nivel terciario son mayores en las provincias más desarrolladas que en aquellas menos desarrolladas. Como resultado, la educación terciaria presentó un efecto positivo y significativo en el crecimiento de las provincias más desarrolladas, al contrario de la educación primaria y secundaria, cuyos resultados fueron no significativos y negativos. En este sentido, si bien la formación superior en China ha sido responsable de avances significativos en el crecimiento económico, la reducción de las disparidades regionales está condicionada a inversiones en todos los niveles, especialmente en la mejora de la educación primaria y secundaria.

En esta línea de pensamiento, otros estudios indican resultados similares. Entre ellos se destacan las contribuciones de Vandenbussche, Aghion y Meghir (2006), Aghion (2008), Ang, Madsen e Islam (2011), Ott y Soretz (2011), Basu y Mehra (2014) y Madsen (2014).

Gennaioli y otros (2013) estudiaron los efectos del capital humano en el desarrollo en una muestra de más de 1.500 regiones en 110 países del mundo. La muestra comprendía el 74\% de la superficie del planeta y representaba el $97 \%$ del PIB mundial. Los resultados del estudio indicaron que las disparidades regionales están ampliamente influenciadas por las diferencias de capital humano, pero que esas divergencias son más susceptibles a los distintos tipos y niveles de educación. Esas diferencias incluyen desigualdades en materia de calidad y resultan afectadas por la composición del capital humano (calidad del capital humano).

Desde esta perspectiva, los países necesitan un tipo de capital humano específico altamente calificado para sostener el proceso de convergencia económica, de manera que dicho proceso pueda acelerarse en los países donde la participación de estudiantes de alto rendimiento en las escuelas y universidades se vuelve significativa. Estos avances dependen de políticas que interiorizan composiciones distintas en la asignación de los recursos, que están destinados a impulsar los indicadores de educación en sus diferentes niveles (Hanushek y Woessmann, 2012; Ang, Madsen e Islam, 2014).

Si bien esta composición se refleja claramente en las diferentes tasas de crecimiento de los países, algunos aspectos importantes como la migración de mano de obra pueden explicarse por las fluctuaciones en la proximidad con respecto a la frontera. Según Di Maria y Lazarova (2012), muchos estudios sobre migración subrayan sus efectos en la acumulación del nivel, pero no tienen en cuenta su influencia en la composición del capital humano. Esto deriva de las diferencias entre los salarios y la empleabilidad de la mano de obra, que afectan los incentivos y están condicionadas por el grado de desarrollo tecnológico. Las economías situadas en la frontera tecnológica tienen una influencia significativa en la racionalidad económica de los talentos que emigran de las economías en desarrollo. Este efecto distributivo del capital humano altamente calificado entre las diferentes economías supone una clara consecuencia en los niveles y la composición del capital humano, reduciendo la tasa de crecimiento de la productividad en los países y regiones de origen y aumentándola en las economías de destino (Di Maria y Stryszowski, 2009).

Estos resultados pueden explicar la manera en que la expansión de la mano de obra calificada en las regiones menos desarrolladas contribuye proporcionalmente menos a la acumulación del ingreso. Esto 
indica la inexistencia de restricciones institucionales a la migración de mano de obra entre los municipios y estados de la federación, al contrario de la regulación observada entre los países (Hendricks, 2002).

En este orden de ideas, Korpi y Clark (2015) analizaron los datos familiares de una muestra de 982.179 personas, incluidos 126.233 migrantes internos, en los microdatos de Suecia 6 . Los resultados indicaron que los trabajadores más calificados se apropian de la mayor parte de las ganancias significativas en el ingreso, en la medida en que migran a las metrópolis más desarrolladas. Esto sugiere que el capital humano presenta un patrón selectivo en el proceso de migración interna entre las regiones, atribuyendo pesos diferentes en su composición conforme a distintas distribuciones en el ingreso.

\section{Limitaciones del estudio y sugerencias para futuras investigaciones}

Si bien los resultados del ejercicio empírico son convergentes con las alusiones presentadas en el modelo teórico, es necesario presentar adecuadamente algunos aspectos relevantes destacados en el estudio.

En el mercado de trabajo brasileño, el sector público ejerce una notable influencia, incrementando la prima de calificación de la mano de obra y compitiendo con importantes segmentos del sector privado, especialmente en los municipios de menores ingresos. Esto permitió sostener, parcialmente, la expansión del estado de bienestar social por medio del empleo público (Mattos, 2015 y 2011). A esto se suma que, en el Brasil, algunas incoherencias en el concepto de calificación del trabajador asociado al nivel de escolaridad han presentado divergencias en muchas pruebas empíricas, sugiriendo nuevas formas de concepción de la calificación de la mano de obra. Esto se debe a que algunos importantes resultados indican la absorción de trabajadores calificados en ocupaciones que requieren un nivel de educación inferior. Los datos de la PNAD de 1981 a 2001 indican una tendencia a la "precarización" de las habilidades asociada a la subutilización de los trabajadores con mayores niveles de escolaridad en la realidad brasileña. En este caso, un posible excedente de oferta de trabajadores con mayor calificación termina siendo "obligado" a realizar actividades que requieren niveles de calificación inferiores, posiblemente debido a la falta de opciones en el mercado de trabajo (Machado, Oliveira y Carvalho, 2004).

No obstante, en algunas investigaciones recientes se indica que la relación entre las medias salariales de los trabajadores que completaron la educación secundaria presenta una variación de entre 2,65 y 2,9 veces a partir de la década de 2000, que señala un aumento considerable en la demanda de educación superior a lo largo del período (Insper, 2016; Rocha y otros, 2017). Algunas estimaciones realizadas de 2000 a 2010 a nivel municipal indican que una variación de 1 punto porcentual en la proporción de adultos con educación superior está asociada a un aumento de 0,4 puntos porcentuales en la tasa de ocupación y a un crecimiento del 0,9\% de los salarios y del 1,3\% del ingreso per cápita del hogar (Rocha y otros, 2017, pág. 66).

Aunque estos resultados son coherentes con las realidades económicas de distintos períodos, no se ha llegado a un consenso sobre los efectos del capital humano en el desarrollo del Brasil. Los resultados son heterogéneos y deben perfeccionarse en futuras investigaciones para captar con mayor precisión posibles divergencias entre la demanda y la oferta en el mercado de trabajo y sus consecuencias en el crecimiento de los municipios brasileños.

\footnotetext{
6 Base de datos Microdata Online Access (MONA) de Statistics Sweden [en línea] https://www.scb.se/en/services/guidance-forresearchers-and-universities/mona--a-system-for-delivering-microdata/.
} 
La presente investigación indica, en el modelo teórico, la existencia de distintas actividades que requieren factores de producción diferenciados. Desde una perspectiva schumpeteriana, diferentes actividades requieren factores que se vuelven complementarios al patrón tecnológico vigente. En consecuencia, no es razonable tratar diferentes categorías de ocupación con una medida única, según se desprende de relevantes investigaciones nacionales (Machado, Oliveira y Carvalho, 2004; Insper, 2016; Rocha y otros, 2017) e internacionales (Ang, Madsen e Islam, 2011; Ott y Soretz, 2011; Basu y Mehra, 2014; Madsen, 2014).

Asimismo, el análisis de regresión y otras técnicas basadas en la media de la distribución condicional del modelo pueden no captar esas imperfecciones en el mercado de trabajo, sobrestimando o subestimando las relaciones entre las variables. En la técnica propuesta en este estudio, se adoptan las diferencias de ingreso entre los municipios para captar posibles distorsiones de la demanda entre las regiones. Estas distorsiones pueden influir significativamente en el efecto de la mano de obra con educación superior en el ingreso per cápita de los municipios. Además, de no controlarse la endogeneidad, los parámetros estimados pueden reflejar relaciones erróneas que deben interpretarse con la debida precaución (Imbens, 2014; Fan y Liao, 2014; Ackerberg y otros, 2014; Lenkei, Mustafa y Vecchi, 2018; Chaudhuri y Guilkey, 2016).

Además de estos aspectos destacados, cabe subrayar los problemas que derivan de la utilización de una base de datos agregados. En este caso, según el nivel de agregación, mucha información sobre las variables puede "suavizar" las relaciones en el modelo, limitando potencialmente los resultados. Sin embargo, los potenciales costos mencionados se minimizan en la medida en que el modelo teórico expuesto diferencia el ejercicio empírico de los estudios presentados. Si bien no se elude la relevancia de tales restricciones, se hace hincapié en que el enfoque alternativo aquí propuesto atenúa sus costos y los resultados encontrados concuerdan con investigaciones internacionales como las de Vandenbussche, Aghion y Meghir (2006), Ang, Madsen e Islam (2011), Basu y Mehra (2014) y Madsen (2014).

\section{Consideraciones finales}

En el presente estudio, se analizó la contribución del aumento de los trabajadores ocupados con educación superior en el ingreso per cápita de los municipios brasileños, en la medida en que los diferentes ingresos entre los ocupados afectan la magnitud de esta contribución. A partir de un modelo de crecimiento schumpeteriano, basado en los enfoques de Aghion y Howitt (1998 y 2009), la decisión de la empresa monopolista para maximizar sus beneficios depende de dos componentes del capital humano: i) trabajadores altamente calificados dedicados a actividades de innovación y ii) trabajadores poco calificados encargados de actividades de imitación y transferencia tecnológica.

En condiciones de optimización, la demanda de mano de obra poco calificada de la empresa depende positivamente del grado de proximidad con respecto a la frontera tecnológica. A partir de los resultados del modelo, las empresas más distantes del líder tecnológico del sector (frontera) presentan mayor peso en la composición de su demanda final de trabajadores poco calificados. A medida que la empresa se acerca a la frontera tecnológica, la demanda total atribuye mayor peso a la contratación de trabajadores dedicados a actividades de innovación y, por lo tanto, con mayores niveles de calificación. En las diferencias salariales entre los factores, la distancia de la frontera aumenta los costos de oportunidad del trabajo calificado reduciendo la prima de calificación, como sugieren los resultados del modelo empírico.

Los resultados empíricos a partir de los datos de los municipios del censo de 2010 indican que la contribución del aumento de los ocupados con educación superior tiende a ser mayor en los municipios con menores diferencias de ingreso en relación con el mayor valor registrado en cada 
unidad federativa. El aumento de las diferencias de ingreso eleva los costos de oportunidad del factor, contribuyendo a un posible efecto migratorio en las regiones con mayores ingresos. Los resultados también sugieren que los municipios con bajos ingresos per cápita presentan menores oportunidades y, por consiguiente, menores beneficios asociados a una demanda reprimida.

Los municipios con ingresos superiores al $46 \%$ del máximo ingreso registrado en el estado presentan una relación positiva entre el aumento de ocupados con educación superior y el ingreso per cápita. Estos resultados indican que el efecto final de la expansión de la mano de obra calificada en el país depende de características importantes del mercado de trabajo que aumentan la demanda de las competencias adquiridas en la educación superior. El descuido de políticas públicas orientadas tanto a la oferta (formación de la educación superior) como a la demanda (ocupación en actividades generadoras de ingresos para este tipo de factor) puede contribuir al aumento de las desigualdades regionales.

Si bien estos resultados señalan una mejor articulación entre las políticas (en especial, educativas e industriales), las limitaciones del estudio sugieren futuras líneas de investigación: diferentes tipos de formación en la educación superior, calidad de la formación, diferencias entre la educación pública y privada, diferencias entre las inversiones por niveles (primario, secundario y superior), entre otros factores relevantes que quedaron fuera del alcance de esta investigación.

Además, la adhesión de los resultados del ejercicio empírico al modelo teórico requiere una interpretación que aún debe hacerse con la debida cautela. En este caso, una parte del aumento de los ingresos en los municipios puede estar influida por actividades que emplean recursos humanos calificados cuando, en realidad, necesitan recursos menos calificados. Por lo tanto, en el presente estudio se señala la necesidad de diferentes tipos de capital humano para distintas actividades, que pueden repercutir en patrones de crecimiento diferenciado entre las economías.

Si bien este crecimiento diferenciado entre las economías puede explicar parte de las desigualdades, se necesita una mayor profundización, especialmente en los diferentes mercados de trabajo, para comprender cómo y qué tipos de recursos humanos son más necesarios según las características de los municipios. Por último, los resultados presentados indican que el concepto de capital humano, a partir de métricas agregadas, puede constituir un factor limitante a la manera en que la educación puede contribuir al desarrollo.

\section{Bibliografía}

Acemoglu, D. (1998), "Why do new technologies complement skills? Directed technical change and wage inequality", The Quarterly Journal of Economics, vol. 113, № 4, noviembre.

Acemoglu, D., F. Gallego y J. Robinson (2014), "Institutions, human capital and development", Annual Review of Economics, vol. 6.

Acharya, V., R. Baghai y K. Subramanian (2014), "Wrongful discharge laws and innovation", The Review of Financial Studies, vol. 27, N 1, enero.

Ackerberg, D. y otros (2014), "Asymptotic efficiency of semiparametric two-step GMM", The Review of Economic Studies, vol. 81, № 3, julio.

Aghion, P. (2008), "Higher education and innovation", Perspektiven der Wirtschaftspolitik, vol. 9, № S1, mayo.

Aghion, P. y P. Howitt (2009), The Economics of Growth, Cambridge, The MIT Press. (1998), Endogenous Growth Theory, Cambridge, The MIT Press.

Aghion, P. y otros (2009), "The causal impact of education on economic growth: evidence from the United States", Brookings Papers on Economic Activity, marzo, Washington, D.C., Brookings Institution Press.

Ang, J., J. Madsen y M. Islam (2014), "Quality-adjusted human capital and productivity growth", Economic Inquiry, vol. 52, $\mathrm{N}^{\circ} 2$, abril.

(2011), "The effects of human capital composition on technological convergence", Journal of Macroeconomics, vol. $33, N^{\circ} 3$. 
Barro, R. (2001), "Human capital and growth", The American Economic Review, vol. 91, № 2.

Barro, R. y X. Sala-i-Martin (1992), "Convergence", Journal of Political Economy, vol. 100, № 2.

Bastgen, A. y C. Holzner (2017), "Employment protection and the market for innovations", Labour Economics, vol. 46, junio.

Basu, S. y M. Mehra (2014), "Endogenous human capital formation, distance to frontier and growth", Research in Economics, vol. 68, № 2, junio.

Baum, C., M. Schaffer y S. Stillman (2003), "Instrumental variables and GMM: estimation and testing", The Stata Journal, vol. 3, No 1, Texas, Stata Corporation.

Becker, S., E. Hornung y L. Woessmann (2011), "Education and catch-up in the Industrial Revolution", American Economic Journal: Macroeconomics, vol. 3, No 3, julio.

Becker, S. y L. Woessmann (2009), "Was Weber wrong? A human capital theory of protestant economic history", The Quarterly Journal of Economics, vol. 124, № 2, mayo.

Benhabib, J. y M. Spiegel (2005), "Human capital and technology diffusion", Handbook of Economic Growth, vol. 1A, P. Aghion y S. Durlauf (eds.), Ámsterdam, North-Holland.

(1994), "The role of human capital in economic development: evidence from aggregate cross-country data", Journal of Monetary Economics, vol. 34, № 2, octubre.

Bond, S., A. Hoeffler y J. Temple (2001), "GMM estimation of empirical growth models", Economics Discussion Papers, N ${ }^{\circ}$ 2001-W21, Oxford, Universidad de Oxford.

Cameron, A. y P. Trivedi (2005), Microeconometrics: Methods and Applications, Nueva York, Cambridge University Press.

Caselli, F. y W. Coleman (2006), "The world technology frontier", The American Economic Review, vol. 96, $N^{\circ} 3$, junio.

Chaudhuri, S. y D. Guilkey (2016), "GMM with multiple missing variables", Journal of Applied Econometrics, vol. $31, N^{\circ} 4$.

Costa, D., A. Costa y F. Barbosa (2013), "Financiamento público e expansão da educação superior federal no Brasil: o REUNI e as perspectivas para o REUNI 2", Revista Gestão Universitária na América Latina, vol. $6, \mathrm{~N}^{\circ} 1$, enero.

Cury, C. (2014), "Formação e conhecimento: perspectivas filosóficas e sociológicas", Avaliação: Revista da Avaliação da Educação Superior, vol. 19, № 3.

Di Maria, C. y E. Lazarova (2012), "Migration, human capital formation, and growth: an empirical investigation", World Development, vol. 40, № 5, mayo.

Di Maria, C. y P. Stryszowski (2009), "Migration, human capital accumulation and economic development", Journal of Development Economics, vol. 90, № 2, noviembre.

Dosi, G., G. Fagiolo y A. Roventini (2010), "Schumpeter meeting Keynes: a policy-friendly model of endogenous growth and business cycles", Journal of Economic Dynamics and Control, vol. 34, № 9.

Durlauf, S., P. Johnson y J. Temple (2005), "Growth econometrics", Handbook of Economic Growth, vol. 1A, P. Aghion y S. Durlauf (eds.), Ámsterdam, North-Holland.

Fan, J. e Y. Liao (2014), "Endogeneity in high dimensions", The Annals of Statistics, vol. 42, № 3, junio.

Felicetti, V., A. Cabrera y M. Costa-Morosini (2014), "Aluno ProUni: impacto na institução de educação superior e na sociedade", Revista Iberoamericana de Educación Superior, vol. 5, № 13.

Galvao, A. y G. Montes-Rojas (2015), "On the equivalence of instrumental variables estimators for linear models", Economics Letters, vol. 134, septiembre.

Gennaioli, N. y otros (2013), "Human capital and regional development", The Quarterly Journal of Economics, vol. 128, № 1, Oxford, Oxford University Press.

Greene, W. (1999), Análisis econométrico, 3.a edición, Madrid, Prentice Hall.

Hansen, L. (1982), "Large sample properties of generalized method of moments estimators", Econometrica, vol. 50, N 4, julio.

Hanushek, E. y L. Woessmann (2012), "Do better schools lead to more growth? Cognitive skills, economic outcomes, and causation", Journal of Economic Growth, vol. 17, № 4, diciembre.

Hayashi, F. (2000), Econometrics, Princeton, Princeton University Press.

Hendricks, L. (2002), "How important is human capital for development? Evidence from immigrant earnings", The American Economic Review, vol. 92, № 1, marzo.

Hsiao, C. y J. Zhang (2015), "IV, GMM or likelihood approach to estimate dynamic panel models when either N or $T$ or both are large", Journal of Econometrics, vol. 187, $\mathrm{N}^{\circ} 1$, julio. 
IBGE (Instituto Brasileño de Geografía y Estadística) (s/fa), "Censo demográfico" [en línea] https://www.ibge. gov.br/estatisticas/sociais/populacao/9662-censo-demografico-2010.html?edicao=9673\&t=downloads.

_ (s/fb), "Pesquisa Nacional por Amostra de Domicílios - PNAD" [en línea] https://www.ibge.gov.br/ estatisticas/sociais/populacao/9127-pesquisa-nacional-por-amostra-de-domicilios.html?=\&t=downloads.

Imbens, G. (2014), "Instrumental variables: an econometrician's perspective", Statistical Science, vol. 29, $N^{\circ} 3$, agosto.

INEP (Instituto Nacional de Estudios e Investigaciones Educativas Anísio Teixeira) (2016), "Sinopse Estatística da Educação Superior 2015" [base de datos en línea] http://portal.inep.gov.br/web/guest/sinopsesestatisticas-da-educacao-superior.

Insper (2016), "Panorama do mercado de trabalho" [en línea] https://www.insper.edu.br/wp-content/ uploads/2018/09/Panorama-do-Mercado-de-Trabalho-CPP-2016.pdf.

Kleibergen, F. y R. Paap (2006), "Generalized reduced rank tests using the singular value decomposition", Journal of Econometrics, vol. 133, N 1.

Koeniger, W. (2005), "Dismissal costs and innovation", Economics Letters, vol. 88, № 1, julio.

Korpi, M. y W. Clark (2015), "Internal migration and human capital theory: to what extent is it selective?", Economics Letters, vol. 136, noviembre.

Lenkei, B., G. Mustafa y M. Vecchi (2018), "Growth in emerging economies: is there a role for education?", Economic Modelling, vol. 73, junio.

Lima, K. (2012), "Expansão e reestruturação das universidades federais e intensificação do trabalho docente: o Programa REUNI", Revista de Políticas Públicas, vol. 16, octubre.

Lucas, R. (2009), "Ideas and growth", Economica, vol. 76, № 301, febrero.

Machado, A., A. Oliveira y N. Carvalho (2004), "Tipologia de qualificação da força de trabalho: uma proposta a partir da noção de incompatibilidade entre ocupação e escolaridade", Nova Economia, vol. 14, № 2.

Madsen, J. (2014), "Human capital and the world technology frontier", The Review of Economics and Statistics, vol. 96, № 4, octubre.

Mancebo, D., A. Vale y T. Martins (2015), "Políticas de expansão da educação superior no Brasil 1995-2010", Revista Brasileira de Educação, vol. 20, № 60.

Mattos, F. (2015), "Trajetória do emprego público no Brasil desde o início do século XX", Ensaios FEE, vol. 36, № 1, Porto Alegre, Fundación de Economía y Estadística (FEE). (2011), "Emprego público nos países desenvolvidos: evolução histórica e diferenças nos perfis”, Texto para Discussão, № 1578, Brasilia, Instituto de Investigación Económica Aplicada (IPEA).

Ott, I. y S. Soretz (2011), "Public policies and convergence", Journal of Economic Dynamics and Control, vol. 35, No 9 .

Philippon, T. y A. Reshef (2012), "Wages and human capital in the U.S. finance industry: 1909-2006", The Quarterly Journal of Economics, vol. 127, № 4, noviembre.

PNUD/IPEA/FJP (Programa de las Naciones Unidas para el Desarrollo/Instituto de Investigación Económica Aplicada/Fundación João Pinheiro) (2013), "Atlas de Desarrollo Humano en Brasil” [base de datos en línea] http://www.atlasbrasil.org.br/2013/es/consulta/.

Rocha, R. y otros (2017), "A relação entre o ensino superior público e privado e a renda e emprego nos municípios brasileiros", Pesquisa e Planejamento Econômico, vol. 47, Nㅜㄹ, diciembre.

Schiopu, I. (2015), "Technology adoption, human capital formation and income differences", Journal of Macroeconomics, vol. 45, septiembre.

Vandenbussche, J., P. Aghion y C. Meghir (2006), "Growth, distance to frontier and composition of human capital", Journal of Economic Growth, vol. 11, № 2, junio.

Zhang, C. y L. Zhuang (2011), "The composition of human capital and economic growth: evidence from China using dynamic panel data analysis", China Economic Review, vol. 22, № 1, marzo. 